Cahiers québécois de démographie

\title{
Fécondité et travail féminin en Grèce': un modèle micro-économique
}

A MICRO-ECONOMIC MODEL FOR THE FERTILITY-EMPLOYMENT RELATIONSHIP IN GREECE FECUNDIDAD Y TRABAJO FEMENINO EN GRECIA': MODELO MICRO-ECONOMICO

\section{Hariclia Symeonidou et Michael Magdalinos}

Volume 22, numéro 2, automne 1993

URI : https://id.erudit.org/iderudit/010149ar

DOI : https://doi.org/10.7202/010149ar

Aller au sommaire du numéro

Éditeur(s)

Association des démographes du Québec

ISSN

0380-1721 (imprimé)

1705-1495 (numérique)

Découvrir la revue

Citer cet article

Symeonidou, H. \& Magdalinos, M. (1993). Fécondité et travail féminin en

Grèce': un modèle micro-économique. Cahiers québécois de démographie, 22(2), 285-312. https://doi.org/10.7202/010149ar

\section{Résumé de l'article}

Magdalinos et Symeonidou (1989) ont étudié la relation entre la fécondité et l'emploi chez les femmes de la région métropolitaine d'Athènes. L'analyse présentée ici porte sur le reste de la Grèce. Elle fait appel à un échantillon de 3193 femmes mariées comprenant 1532 femmes des régions urbaines (à l'exclusion d'Athènes) et 1661 femmes des régions rurales. Un test de détection des erreurs de spécification a mis en évidence des différences de structure entre les deux univers ainsi que l'hétéroscédasticité de l'équation d'offre pour le travail et de l'équation de demande pour les enfants. Un dernier modèle composé de cinq équations de structure et de quatre équations de variance a été estimé. Ces nouveaux résultats confirment les précédents : l'emploi influence la fécondité directement et à travers les attitudes liées aux rôles féminins. On ne note aucune influence directe significative de la fécondité sur l'emploi, mais dans les zones rurales on observe un effet indirect significatif, par l'intermédiaire des attitudes liées aux rôles sexuels. Comme prévu, les équations de fécondité et d'emploi présentent des différences significatives entre zones urbaines et zones rurales.
Tous droits réservés @ Association des démographes du Québec, 1993
Ce document est protégé par la loi sur le droit d'auteur. L'utilisation des services d'Érudit (y compris la reproduction) est assujettie à sa politique d'utilisation que vous pouvez consulter en ligne.

https://apropos.erudit.org/fr/usagers/politique-dutilisation/ 
Cahiers québécois de démographie

Vol. 22, no 2, automne 1993, p. 285-312.

\title{
Fécondité et travail féminin en Grèce : un modèle micro-économique
}

\author{
Hariclia SYMEONIDOU et Michael MAGDALINOS *
}

Dans une précédente analyse micro-économique de la fécondité consacrée aux femmes de la région métropolitaine d'Athènes ${ }^{1}$ (Magdalinos et Symeonidou, 1989), nous avons présenté un système d'équations simultanées où la fécondité, l'emploi et les attitudes à l'égard des rôles féminins étaient déterminés par un ensemble de variables socio-économiques exogènes. Nous avons également modélisé le processus de génération des erreurs pour les équations d'estimation de la fécondité et de l'emploi, afin d'obtenir des procédures d'estimation et de test plus efficaces et de dégager de l'information utile à la décision politique. Ce dernier objectif revêt une importance particulière en Grèce, où le déclin démographique et le vieillissement de la population sont sources de vives inquiétudes (ibid. : 121).

Par la suite, des données sur les femmes des autres régions urbaines et des régions rurales de la Grèce étant devenues disponibles, nous les avons utilisées dans un système similaire d'équations simultanées. De la sorte, il devenait possible de comparer les comportements des femmes des trois milieux

* Respectivement du Centre national de la recherche sociale et de l'École d'économie et de sciences commerciales d'Athènes. Les données présentées dans cet article proviennent d'une enquête nationale sur la fécondité réalisée par le Centre national de la recherche sociale de Grèce sous la direction d'Hariclia Symeonidou. Les auteurs remercient les professeurs C. Langford, de la London School of Economics, ainsi que G. Bitros, T. Kollintzas et K. Prodromides, de l'École d'économie d'Athènes, pour leurs remarques judicieuses. Traduit de l'anglais par Johanne Archambault, sous la supervision de Danielle Gauvreau.

1 Athènes dans la suite du texte. 
(Athènes, autres régions urbaines et régions rurales) et de vérifier si les politiques proposées dans notre étude sur Athènes conviennent au reste du pays.

Dans cet article, nous présenterons successivement les spécifications a priori de notre modèle, l'estimation du coût du loisir, l'estimation et les résultats de quelques tests de détection des erreurs de spécification et enfin l'estimation du modèle final et l'interprétation des résultats. Pour clore, nous examinerons les différences de fécondité et d'emploi entre les trois milieux de vie.

\section{LE MODÈLE}

Nous présumons que chaque femme maximise son utilité, qui est fonction de trois facteurs : le nombre d'enfants, le temps libre (loisir) et un indicateur agrégé de biens de consommation. Cette maximisation se situe dans une double contrainte de budget et de temps; le temps disponible est ainsi décomposé en temps alloué au loisir, temps alloué à l'emploi et temps alloué au travail domestique.

En outre, nous postulons que les variations des préférences, c'est-à-dire la forme de la fonction d'utilité, sont déterminées par les attitudes à l'égard des rôles féminins, ellesmêmes influencées par les décisions touchant le nombre d'enfants et la participation des femmes à la main-d'œuvre.

Sous l'hypothèse que ce comportement dynamique mène à un équilibre à long terme, nous concluons que la fécondité, l'emploi et les attitudes liées aux rôles sexuels sont simultanément déterminés par des variables de revenu et de coût (Magdalinos et Symeonidou, 1989 : 122-124).

Pour compléter l'analyse, nous y avons incorporé des variables démographiques de même que des variables relatives aux antécédents familiaux de la répondante et à son mari. La liste des variables est présentée ci-après; les équations où elles apparaissent comme variables explicatives sont indiquées entre parenthèses ( $F$ désigne l'équation de fécondité, $S$ l'équation de rôles sexuels et $\mathrm{E}$ l'équation d'emploi).

\section{Variables endogènes}

EMPL Emploi : pourcentage des années de mariage au cours desquelles un emploi à plein temps a été 
occupé. Nous n'avions pas les données nécessaires pour tenir compte du travail à temps partiel. L'erreur due à cette omission est probablement négligeable car notre échantillon comprenait un pourcentage infime de femmes travaillant à temps partiel (voir le tableau 1, plus bas). En outre, comme, dans notre échantillon, le nombre d'années de mariage variait entre 5 et 29 , nous avons préféré utiliser comme variable une proportion plutôt que le nombre d'années travaillées durant les années de mariage, afin de mieux exprimer le désir de travailler des femmes ( $F, S)$.

RÔLSEX Attitude des femmes relativement aux rôles sexuels, mesurée à l'aide d'une échelle de Likert à 15 éléments (d'après Smith-Lovin et Tickamyer, 1978). Nous avons calculê un score discriminant sur une échelle de $O$ (attitude très conservatrice) à 100 (contestation) (F, E).

FÉCON Fécondité atteinte : nombre de naissances vivantes au moment de l'enquête. Pour ne pas aboutir à des coefficients de régression trop faibles, nous avons multipliê cette valeur par 100 (S, E).

\section{Variables de revenu et de coûts}

\$LOISIR Coût du loisir, réel ou vraisemblable (raisonnable). Pour un emploi rémunéré à plein temps, ce coût est égal aux gains mensuels réels, en milliers de drachmes. Pour les autres types d'emploi et pour les femmes qui ne travaillaient pas au moment de la cueillette des données, cette variable est prédite au moyen d'une équation qui sera estimée dans la section suivante $(F, E)$.

REVFAM Revenu familial total. Si l'épouse occupe un emploi rémunéré à plein temps à l'extérieur ou dans l'entreprise familiale, cette variable est égale au revenu familial mensuel déclaré, en milliers de drachmes. Si l'épouse ne travaille pas, le revenu familial déclaré est accru d'un montant égal à \$LOISIR. Sỉ l'épouse travaille à temps partiel, le revenu déclaré est accru d'un montant égal à $(1$ - 40/HEU $) \times$ \$LOISIR, où HEU représente le nombre d'heures de 
travail par semaine et 40 le nombre d'heures correspondant à un emploi à plein temps (F, E).

\$RELOISIR Coût relatif du loisir. Cette variable est égale au ratio (\$LOISIR/REVFAM) $\times 100$; (S, E).

NPIĖCES Nombre de pièces possédées. La variable est égale à 0 si le ménage n'est pas propriétaire, au nombre de pièces de la maison du ménage dans le cas contraire $(\mathrm{F}, \mathrm{E})$.

SCOLAR Nombre d'années de scolarité de la répondante (F, E, S).

SCOLARH Nombre d'années de scolarité du mari de la répondante $(\mathrm{F}, \mathrm{E})$.

SSÉ Statut socio-économique de la famille, représenté par le prestige professionnel du mari (Treiman, 1977 : 235-260) (F, E, S).

AIDE Aide de la part de parents. La variable vaut 1 si la répondante dit avoir reçu beaucoup d'aide de sa parenté pour le soin des enfants ou le ménage, 0 dans le cas contraire $(F, E)$.

\section{Variables prédéterminées}

EMPLAV Emploi avant le mariage. Nombre d'années travaillées avant le mariage ( $\mathrm{E}, \mathrm{S})$.

ENFDÉS Fécondité souhaitée (nombre d'enfants désiré) par la répondante au moment de son mariage (F).

\section{Variables démographiques}

ÂGE Âge de la répondante au moment de l'enquête (E, S).

DURMAR Nombre d'années de mariage (S, F).

ÂGEMAR Âge de la répondante au mariage (F).

ORIG Région d'origine. La variable vaut 1 si, jusqu'à l'âge de 15 ans, la rêpondante a passé la plus grande partie de sa vie en région rurale, 0 dans le cas contraire (E, S, F).

RÉSID Région de résidence. La variable est codée 1 si la famille habite une région rurale, 0 autrement (E, S, F). 


\section{Antêcédents familiaux}

TRAVMÈ Mère active. Variable codée 1 si la mère de la répondante a occupé un emploi à un moment quelconque, 0 dans le cas contraire $(\mathrm{E}, \mathrm{S})$.

ENFME Nombre d'enfants de la mère de la répondante (F).

SCOLMẼ Nombre d'années de scolarité terminées par la mère de la répondante (S).

SCOLPÈ Nombre d'années de scolarité terminées par le père de la répondante (S).

SSÉPÈ Statut socio-économique du père de la répondante, mesuré de la même manière que SSÉ (S).

\section{Contrôle du mari}

VETOH Interdiction du mari. Prend la valeur de 1 si le mari ne permet pas à sa femme de travailler, 0 autrement $(\mathrm{E})$.

ORIGH Région d'origine du mari. Codifiée de la même manière qu'ORIG $(\mathrm{E}, \mathrm{S}, \mathrm{F})$.

ENFMÈH Nombre d'enfants de la mère du mari $(\mathrm{F})$.

On notera que, dans ce modèle, les variables \$LOISIR, REVFAM et \$RELOISIR remplacent les variables REVSÉP (revenu familial sans le salaire de l'épouse) et \$OPT (coût d'option du travail) utilisées dans Magdalinos et Symeonidou (1989).

Ce changement, en partie motivé par des raisons théoriques (REVFAM représente la véritable contrainte de budget dans la maximisation de l'utilité), vise aussi à permettre d'observer les effets de REVFAM et de \$LOISIR sur la fécondité (l'on s'attend à ce que ces effets soient opposés). La décision de travailler est influencée par \$RELOISIR, qui est également une mesure de l'autonomie financière (potentielle) de la femme et fait donc partie de l'équation de rôles sexuels.

Les raisons de l'inclusion des autres variables dans les trois équations, manifestes, sont expliquées dans Magdalinos et Symeonidou (1989 : 125-127). De nouveau, il faut éviter de faire apparaitre ensemble dans la même équation les variables ÂGE, DURMAR et ÂGEMAR, parfaitement colinéaires. 


\section{DONNÉES ET MESURE}

Les données dont nous nous sommes servis pour cette analyse proviennent de l'Enquête nationale sur la fécondité réalisée par le Centre national de la recherche sociale de Grèce (EKKE) entre novembre 1984 et février 1985, sur un échantillon stratifié de 4560 femmes nées entre 1939 et 1969, qui en étaient à leur première union et vivaient dans une région urbaine (Athènes exceptée) ou dans une région rurale. Notre sous-échantillon comprend 3193 femmes, après élimination des répondantes qui :

- connaissaient des problèmes de fertilité et risquaient de ne pas pouvoir se reproduire durant leur vie matrimoniale;

- avaient refusé de répondre ou passé outre à au moins l'une des questions relatives aux variables définies ci-dessus;

- comptaient moins de cinq ans de mariage (afin que toutes les femmes de l'échantillon aient, minimalement, eu la chance de commencer à mettre en ouvre leurs projets de travail et de maternitê).

La première étape de la construction de notre modèle consistait à formuler une équation de taux de salaire afin de calculer ensuite le coût du loisir pour les femmes qui ne travaillaient pas. La structuration de l'échantillon par région eu égard aux situations d'emploi apparaît au tableau 1 . On note que seulement $4,6 \%$ des femmes travaillaient à temps partiel (moins de 22 heures par semaine) et que, dans les régions rurales, la majorité des femmes employées dans une entreprise familiale n'étaient pas rémunérées (selon l'enquête, 92,9\% de

TABLEAU 1 - Répartition des femmes de l'échantillon selon le lieu de résidence et la situation de travail (\%)

\begin{tabular}{|c|c|c|c|}
\hline & $\begin{array}{l}\text { Régions } \\
\text { urbaines }\end{array}$ & $\begin{array}{l}\text { Régions } \\
\text { rurales }\end{array}$ & Total \\
\hline Taille de l'échantillon (N) & 1532 & 1661 & 3193 \\
\hline Ne travaillent pas & 67,3 & 45,7 & 56,1 \\
\hline $\begin{array}{l}\text { Travaillent à temps partiel } \\
\text { dans l'entreprise familiale }\end{array}$ & 1,0 & 2,0 & 1,5 \\
\hline Salariées, à temps partiel & 4,0 & 2,2 & 3,1 \\
\hline $\begin{array}{l}\text { Travaillent à plein temps } \\
\text { dans l'entreprise familiale }\end{array}$ & 4,6 & 34,7 & 20,3 \\
\hline Salariêes, à plein temps & 23,2 & 15,4 & 19,1 \\
\hline
\end{tabular}


ces entreprises étaient des fermes). Nous avons utilisé un échantillon de 708 femmes $(22,2 \%)$ pour calculer l'équation salariale.

On sait que les estimations issues d'échantillons dëfinis par un critère entraînant une autosélection des répondants ${ }^{2}$ peuvent comporter un biais, à cause des corrélations entre les variables explicatives et l'erreur stochastique produite par la règle d'échantillonnage. Pour corriger ce biais, nous avons utilisé la méthode proposée par Olsen (1980), dont la première étape est l'estimation d'une équation de participation à la main-d'œuvre, à l'aide du modèle de probabilité linéaire suivant :

$$
\begin{aligned}
& \mathrm{y}=0,04202+0,02483 \text { SCOLAR }-0,01937 \text { RÉSID } \\
& (0,002) \quad(0,016) \\
& \text { + 0,11946 AIDE + 0,04025 TRAVME - 0,23435 VETOH } \\
& (0,014) \quad(0,016) \quad(0,026) \\
& -0,06876 \mathrm{ENF}_{0-2}-0,04537 \mathrm{ENF}_{2-6}-0,00373 \mathrm{ENF}_{>6} \\
& \begin{array}{lll}
(0,024) & (0,012) & (0,008)
\end{array} \\
& +0,12817 \text { HSSEMPL - 0,12887 HAGR - 0,10568 HNAGR } \\
& (0,037) \quad(0,019) \quad[0,025)
\end{aligned}
$$

$$
\mathrm{R}^{2}=0,122 \quad \mathrm{R}^{2} \text { ajusté }=0,119 \quad \mathrm{~s}^{2}=0,15 \quad \mathrm{~F}=40,22
$$

où y est une variable muette prenant la valeur de 1 si l'êpouse occupait un emploi rémunéré, de 0 dans le cas contraire. Les variables SCOLAR, RÉSID, AIDE, TRAVMÈ et VETOH ont été définies ci-dessus. $\mathrm{ENF}_{0-2}, \mathrm{ENF}_{2-6}$ et $\mathrm{ENF}_{>6}$ représentent les enfants de moins de 2 ans, de 2 ans à 6 ans et de plus de 6 ans (respectivement) vivant avec leurs parents. HSSEMPL, HAGR et HNAGR sont des variables muettes qui prennent la valeur de 1 si le mari était sans emploi ou dirigeait une entreprise agricole ou non agricole (respectivement). Les écarts types sont indiqués sous les coefficients.

À partir du modèle de probabilité linéaire, il était possible d'établir le facteur de correction :

$$
\mathrm{FC}=(\hat{\mathrm{y}}-1) / 3
$$

et de calculer l'équation des salaires. La variable dépendante est le logarithme naturel du salaire mensuel réel en milliers de

2 NDLT: self-selected samples (par exemple, les femmes actives le sont en partie par choix). 
drachmes; les résultats de l'estimation sont présentés au tableau 2. Les variables RÉSID et SSÉ ont été définies plus haut; EXP représente le nombre d'années d'expérience de travail (avant et après le mariage). HEUTRAV correspond au nombre d'heures de travail par semaine et SCOL1, SCOL2 et SCOL3 sont des variables muettes égales à 1 si l'épouse a (respectivement) terminé les neuf années d'études obligatoires, fini ses études secondaires ou acquis une formation professionnelle équivalente, ou fait des études universitaires ou des études supérieures équivalentes. Les écarts types apparaissent sous les coefficients.

Le facteur de correction n'est pas significatif et les autres coefficients ne présentent que des changements infimes. On peut en conclure que le biais lié à la sélection est négligeable. C'est pourquoi nous avons utilisé l'équation non corrigée, avec travail à plein temps (HEUTRAV $=40$ ), pour prédire le coût du

TABLEAU 2-Équation des taux de salaire avec et sans correction de l'erreur due à la sélection

\begin{tabular}{lcc}
\hline $\begin{array}{l}\text { Variables } \\
\text { explicatives }\end{array}$ & Équation corrigée & Équation non corrigée \\
\hline RÉSID & $-0,14609$ & $-0,15384$ \\
& $(0,037)$ & $(0,036)$ \\
SSÉ & 0,00226 & 0,00237 \\
& $(0,001)$ & $(0,001)$ \\
EXP & 0,00323 & 0,00329 \\
& $(0,002)$ & $(0,002)$ \\
HEUTRAV & 0,01331 & 0,01326 \\
& $(0,001)$ & $(0,001)$ \\
SCOL1 & 0,18756 & 0,20258 \\
& $(0,085)$ & $(0,083)$ \\
SCOL2 & 0,21707 & 0,22360 \\
SCOL3 & $(0,092)$ & $(0,092)$ \\
& 0,30403 & 0,31608 \\
Facteur de correction & $(0,060)$ & $(0,058)$ \\
& 0,15397 & - \\
\hline$R^{2}$ & $(0,184)$ & 0,359 \\
$\mathrm{R}^{2}$ ajusté & 0,360 & 0,353 \\
$\mathrm{~s}^{2}$ & 0,352 & 0,188 \\
\hline
\end{tabular}


loisir dans le cas des femmes qui : i) ne travaillaient pas au moment de l'enquête, ii) travaillaient sans salaire dans une entreprise familiale ou iii) travaillaient à temps partiel. Ces prédictions, avec le salaire réel des femmes qui occupaient un emploi rémunéré à plein temps, ont constitué la variable coût du loisir.

Mroz (1987) fait remarquer que le salaire des femmes est une variable endogène. Il en va de même pour les prédictions de taux de salaire, puisqu'elles dépendent des années d'expérience de travail des femmes. Pour contourner ce problème et éliminer le caractère endogène de la variable, nous l'avons estimée à l'aide des variables exogènes de notre modèle (à l'exclusion de REVFAM et de \$RELOISIR, évidemment), et au lieu d'utiliser la variable initiale, nous nous sommes servis de la variable instrumentale correspondante pour calculer les variables REVFAM et \$RELOISIR et pour les trois équations. Notons que la variable instrumentale est en forte corrélation avec la variable initiale : le coefficient, de 0,671, indique que $45 \%$ de la variation initiale est présente dans la variable instrumentale. En outre, l'interprétation conceptuelle des trois variables calculées à partir de cette dernière (SLOISIR, REVFAM, \$RELOISIR) ne change pas puisque cette substitution à la variable initiale est strictement une technique d'estimation.

\section{ESTIMATION ET TEST}

Pour faire les calculs du modèle, nous avons recouru à la méthode des moindres carrés à deux étapes. Les résultats apparaissent au tableau 3. Les équations de fécondité et d'emploi présentent des signes évidents d'erreurs de spécification : EMPL et FÉCON sont en corrélation positive, SCOLAR et SCOLARH n'ont pas le signe attendu, etc.

Avant de pousser plus loin l'analyse du modèle, il y a donc lieu de faire une parenthèse sur les tests de détection des erreurs de spécification. Pour le type de modèle que nous utilisons (statique, estimé à l'aide de micro-données), l'on distingue trois sources d'erreur.

\section{Stabilité des équations}

Le tableau 1 montre clairement que le régime de travail des femmes n'est pas du tout le même dans les régions urbaines et dans les régions rurales. En milieu rural, la majorité des 
TABLEAU 3 - Résultats des estimations par la méthode des moindres carrés à deux étapes

\begin{tabular}{|c|c|c|c|}
\hline Variables Équations & Fécondité & Emploi & Rôles sexuels \\
\hline FÉCON & -1 & $\begin{array}{l}0,014011^{*} \\
(0,023)\end{array}$ & $\begin{array}{l}-0,03079 \\
(0,017)\end{array}$ \\
\hline EMPL & $\begin{array}{l}0,34448^{*} \\
(0,107)\end{array}$ & -1 & $\begin{array}{l}0,08423^{*} \\
(0,032)\end{array}$ \\
\hline RÔLSEX & $\begin{array}{l}-2,6460 \\
(1,748)\end{array}$ & $\begin{array}{l}0,95762^{*} \\
(0,281)\end{array}$ & -1 \\
\hline \$LOISIR & $\begin{array}{l}-0,46485 \\
(0,269)\end{array}$ & $\begin{array}{l}0,9556^{*} \\
(0,12)\end{array}$ & - \\
\hline REVFAM & $\begin{array}{l}0,12478 \\
(0,081)\end{array}$ & $\begin{array}{c}-0,116^{*} \\
(0,042)\end{array}$ & - \\
\hline \$RELOISIR & - & $\begin{array}{l}0,46705^{*} \\
(0,065)\end{array}$ & $\begin{array}{l}0,07818^{*} \\
(0,032)\end{array}$ \\
\hline NPIECES & $\begin{array}{l}-0,46304 \\
(1,01)\end{array}$ & $\begin{array}{c}1,3754^{*} \\
(0,372)\end{array}$ & - \\
\hline SCOLAR & $\begin{array}{l}2,7786 \\
(1,53)\end{array}$ & $\begin{array}{c}-0,39478 \\
(0,597)\end{array}$ & $\begin{array}{l}1,8457^{*} \\
(0,172)\end{array}$ \\
\hline SCOLARH & $\begin{array}{l}0,39747 \\
(0,692)\end{array}$ & $\begin{array}{l}-0,6757^{*} \\
(0,261)\end{array}$ & - \\
\hline SSE & $\begin{array}{l}0,37829^{*} \\
(0,164)\end{array}$ & $\begin{array}{l}0,02322 \\
(0,064)\end{array}$ & $\begin{array}{l}0,09364^{*} \\
(0,034)\end{array}$ \\
\hline AIDE & $\begin{array}{l}1,3671 \\
(3,06)\end{array}$ & $\begin{array}{l}6,4699 * \\
(1,16)\end{array}$ & - \\
\hline EMPLAV & - & $\begin{array}{l}2,455^{*} \\
(0,158)\end{array}$ & $\begin{array}{l}0,05627 \\
(0,132)\end{array}$ \\
\hline ENFDÉS & $\begin{array}{l}18,171 * \\
(1,5)\end{array}$ & - & - \\
\hline ÂGE & - & $\begin{array}{l}0,81715^{*} \\
(0,143)\end{array}$ & $\begin{array}{c}-0,58308^{*} \\
(0,11)\end{array}$ \\
\hline DURMAR & $\begin{array}{l}0,83212^{*} \\
(0,404)\end{array}$ & - & $\begin{array}{l}0,1783 \\
(0,15)\end{array}$ \\
\hline
\end{tabular}

femmes qui travaillent le font sans recevoir de salaire, au sein de l'entreprise agricole familiale; ce type d'emploi est compatible avec le soin des enfants et l'entretien de la maison. En milieu urbain, élever des enfants coûte sans doute plus cher en termes réels et le travail des enfants est moins courant (Schultz, 1969). Il est donc loin d'être sûr que la simple inclusion de la variable RÉSID dans l'équation suffise à prendre en compte toutes les différences entre les deux milieux.

Nous avons testé l'égalité des coefficients de régression entre régions urbaines et régions rurales dans notre modèle. 
TABLEAU 3 (suite)

\begin{tabular}{|c|c|c|c|}
\hline Variables Équations & Fécondité & Emploi & Rôles sexuels \\
\hline ÂGEMAR & $\begin{array}{l}-3,8045^{*} \\
(0,508)\end{array}$ & - & - \\
\hline ORIG & $\begin{array}{c}-13,465^{*} \\
(4,18)\end{array}$ & $\begin{array}{l}6,3802^{*} \\
(1,6)\end{array}$ & $\begin{array}{l}-2,3529^{*} \\
(0,913)\end{array}$ \\
\hline TRAVME & - & $\begin{array}{l}7,0164^{*} \\
(1,32)\end{array}$ & $\begin{array}{c}-1,3761 \\
(0,805)\end{array}$ \\
\hline ENFME & $\begin{array}{c}1,027 \\
(0,754)\end{array}$ & - & - \\
\hline SCOLME & - & - & $\begin{array}{l}0,31299^{*} \\
(0,147)\end{array}$ \\
\hline SCOLPE & - & - & $\begin{array}{l}0,27744^{*} \\
(0,137)\end{array}$ \\
\hline SSÉPÈ & - & - & $\begin{array}{l}0,103394 * \\
(0,04)\end{array}$ \\
\hline VETOH & - & $\begin{array}{c}-24,061^{*} \\
(2,14)\end{array}$ & - \\
\hline ORIGH & $\begin{array}{l}0,12063 \\
(4,29)\end{array}$ & $\begin{array}{l}1,8115 \\
(1,64)\end{array}$ & $\begin{array}{r}-2,0463^{*} \\
(0,8998)\end{array}$ \\
\hline ENFMĖH & $\begin{array}{l}1,8975^{*} \\
(0,667)\end{array}$ & - & - \\
\hline RÉSID & $\begin{array}{l}3,2251^{*} \\
(2,07)\end{array}$ & $\begin{array}{l}8,9249^{*} \\
(1,55)\end{array}$ & $\begin{array}{c}-0,7302 \\
(0,893)\end{array}$ \\
\hline Constante & 258,72 & $-104,24$ & 27,767 \\
\hline $\mathrm{R}^{2}$ & 0,149 & 0,410 & 0,252 \\
\hline $\mathrm{R}^{2}$ ajusté & 0,145 & 0,407 & 0,248 \\
\hline $\mathrm{s}^{2}$ & 5938,5 & 885,47 & 324,87 \\
\hline $\mathrm{F}$ & 32,81 & 129,75 & 71,48 \\
\hline
\end{tabular}

NOTE ${ }^{*}$ : significatif au seuil de $5 \%$ (d'après les écarts types indiqués entre parenthèses, eux-mêmes calculés par la formule habituelle des moindres carrés à deux étapes). $R^{2}$ : selon Carter et Nagar (1977); $\mathrm{s}^{2}$ : erreur quadratique moyenne; $\mathrm{F}$ : variance expliquée divisée par $\mathrm{s}^{2}$. Tirets : restrictions a priori; $-1:$ variable dépendante.

Les statistiques de test et les seuils de signification correspondants (test unilatéral) sont les suivants :

Fécondité : $\quad F=2,222, F(16, \infty)=0,00337$

Emploi : $\quad F=5,509, F(16, \infty)=0,00000$

Rôles sexuels : $F=1,156, F(14, \infty)=0,30244$

Ce résultat entraîne le rejet de l'hypothèse de l'égalité des pentes des équations de fécondité et d'emploi. Une partie de la 
différence peut être attribuée au fait que la structure d'erreur n'est pas la même pour les deux régions; nous avons donc décidé de présenter des estimations de variance distinctes pour chacune.

\section{Erreur de spêcification des variables exogènes}

Il est possible que certaines variables classées comme exogènes recèlent une part d'endogénéité. Il ne s'ensuit pas de problème d'estimation à condition que la corrélation entre les variables exogènes et l'erreur stochastique ne soit pas statistiquement significative. Sargan (1958) a proposé un test général de détection des erreurs de spécification réputé pour sa puissance en pareille situation. Ainsi, quand nous avons utilisé la variable initiale \$LOISIR dans les équations et dans le calcul des variables REVFAM et \$RELOISIR, la statistique du test de Sargan et les valeurs critiques associées étaient :

$\begin{array}{ll}\text { Fécondité : } & \mathrm{s}=33,928, \chi^{2}(5)=0,00000 \\ \text { Emploi : } & \mathrm{s}=41,631, \chi^{2}(5)=0,00000 \\ \text { Rôles sexuels : } & \mathrm{s}=13,346, \chi^{2}(7)=0,06411\end{array}$

Il y a donc erreur de spécification dans les équations de fécondité et d'emploi. Pour remédier à cela, dans les équations et pour le calcul de REVFAM et de \$RELOISIR, nous avons substitué à \$LOISIR la variable instrumentale correspondante. Toutefois, il peut subsister des variables classées exogènes comportant une part d'endogénéité. Ainsi en est-il de NPIËCES, car le travail de l'épouse contribue au capital de la famille, de VETOH, car le nombre d'enfants peut avoir incité le mari à interdire à sa femme de travailler, d'AIDE, car le nombre d'enfants et le travail de l'épouse peuvent avoir influencé la décision de la parenté d'offrir de l'aide, et ainsi de suite. Les statistiques du test de Sargan et les seuils de signification associés sont les suivants :

$\begin{array}{lll}\text { Régions urbaines } & \text { Fécondité : } & \mathrm{s}=5,02, \chi^{2}(4)=0,28525 \\ & \text { Emploi : } & \mathrm{s}=4,34, \chi^{2}(4)=0,36194 \\ \text { Régions rurales } & \text { Fécondité : } & \mathrm{s}=6,29, \chi^{2}(4)=0,17851 \\ & \text { Emploi : } & \mathrm{s}=8,79, \chi^{2}(4)=0,06657\end{array}$

Toutes les régions $\quad$ Rôles sexuels : $s=11,51, \chi^{2}(7)=0,11787$ 
C'est dire que le test ne rejette pas l'hypothèse de l'absence de corrêlation. Nous en concluons que les variables exogènes sont classées correctement.

\section{Hêtéroscédasticité}

Jusqu'ici nous avons présumé que les erreurs stochastiques des équations présentent une variance constante dans tout l'échantillon. Ce postulat peut être vérifié grâce à une modification appropriée ${ }^{3}$ du test de Breush-Pagan (1979). Pour nos équations, les statistiques de test et les seuils de signification sont :

Régions urbaines $\quad$ Fécondité : $\quad B P=257,12, \chi^{2}(20)=0,00000$

$$
\text { Emploi : } \quad B P=139,53, \chi^{2}(20)=0,00000
$$

Régions rurales Fécondité : $\quad \mathrm{BP}=142,10, \chi^{2}(20)=0,00000$

Emploi : $\quad B P=95,42, \chi^{2}(20)=0,00000$

Toutes les régions $\quad$ Rôles sexuels : $\mathrm{BP}=16,40, \chi^{2}(21)=0,74677$

Ce résultat mène au rejet de l'hypothèse d'homoscédasticité dans les équations d'emploi et de fécondité des régions rurales et des régions urbaines.

La théorie statistique nous apprend que s'il y a hẻtéroscédasticité les estimateurs utilisés dans l'approche des moindres carrés à deux étapes ne sont pas efficaces, même asymptotiquement. Surtout, les écarts types estimés ne constituent pas une représentation cohérente des écarts types réels (qu'habituellement ils sous-estiment). La combinaison des deux effets risque fort d'embrouiller et d'invalider toute inférence à partir des données.

\section{AUTRE ESTIMATION}

Pour pallier l'hétéroscédasticité, nous avons le choix entre des méthodes paramétriques (voir par exemple Harvey, 1976; Amemiya, 1977) et des méthodes non paramétriques (voir par

3 Le test statistique devrait être effectué à partir de la forme réduite des résidus partiellement restreints, c'est-à-dire des résidus calculés avec les variables instrumentales plutôt qu'avec les variables explicatives endogènes. Une preuve de la validité du test est disponible auprès des auteurs. 
exemple White, 1980; Robinson, 1987). Utilisées mal à propos, ces dernières ont fait l'objet de critiques, du fait que leur justification est purement asymptotique et potentiellement trompeuse quand on travaille avec des ensembles finis de données (Leamer, 1988). Mais il faut surtout retenir que les méthodes paramétriques supposent le calcul d'une équation de variance dont on peut tirer des informations utiles pour la formulation de politiques.

Pour notre part, nous avons opté pour une modification de la méthode proposée par Amemiya (1977) pour un modèle à équation unique. Cette méthode permet d'obtenir des estimateurs cohérents et asymptotiquement efficaces des coefficients des équations tant de structure que de variance ${ }^{4}$. De plus, les écarts types estimés constituent des estimations cohérentes des écarts types réels, de sorte que le test de signification est possible dans les deux équations. Les tableaux 4 et 5 présentent les résultats de l'estimation des équations de fécondité et d'emploi pour les régions urbaines et les régions rurales (dans le cas des rôles sexuels, l'équation est la même qu'au tableau 3).

En comparant les équations du tableau 3 avec les équations structurelles correspondantes des tableaux 4 et 5 on constate, outre l'augmentation impressionnante des coefficients de détermination 5 , que les secondes sont plus plausibles. Par exemple, dans l'équation de fécondité, les coefficients d'EMPL et de SCOLAR sont négatifs, comme nous nous y attendions.

Les résultats de notre analyse statistique semblent favoriser l'approche sous l'angle "économique". Les variables de base de

4 Les procédures de calcul sont décrites dans Magdalinos et Symeonidou, 1989. Encore ici, il faudrait utiliser la forme réduite des résidus partiellement restreints dans les deux étapes de l'estimation des paramètres de la variance. Une preuve de l'efficacité asymptotique de l'estimateur d'Amemiya pour des estimations basées sur une information limitée est disponible auprès des auteurs.

5 Les $\mathrm{R}^{2}$ doivent être comparés avec prudence. Si nous acceptons l'interprétation traditionnelle qui fait de $\mathrm{R}^{2}$ le pourcentage de la variation de la variable dépendante expliqué par les variables explicatives de la régression, les $R^{2}$ du tableau 3 ne peuvent pas être comparés avec ceux des tableaux 4 et 5 , du seul fait que les variables dépendantes ne sont pas les mêmes $\left(y_{t}\right.$ dans le premier cas et $y_{t} / \hat{\partial}_{t}$ dans le second). D'autre part, si nous acceptons de considérer $\mathrm{R}^{2}$ comme l'estimateur d'un paramètre de la population de base décomposant le modèle en une partie systématique et une partie aléatoire (voir par exemple Carter et Nagar, 1977), alors, les $\mathrm{R}^{2}$ sont comparables, dans le sens que nous préférons le modèle où la part d'aléatoire est relativement moins grande. 
revenu et de coût (\$LOISIR, REVFAM, \$RELOISIR, NPIÈCES, SCOLAR) sont significatives et présentent le signe attendu. L'équation d'emploi en milieu rural est la seule où les variables \$LOISIR et REVFAM ne soient pas significatives, leur effet étanit couvert par celui, significatif, de \$RELOISIR. Dans les deux équations d'emploi et dans l'équation de fécondité pour les régions urbaines, REVFAM est significative et du signe attendu. Mais elle est aussi extrêmement significative dans les équations de variance. À mesure que le revenu total de la famille s'accroît, la liberté de choix de l'épouse augmente également, mais cette liberté accrue ne s'exerce systématiquement dans aucune direction. La scolarité du mari n'est significative que dans l'équation d'emploi pour les régions rurales, où son effet négatif s'explique par la réticence des épouses d'hommes plus instruits à travailler dans le secteur agricole. Pour la même raison, le statut socio-économique de la famille (SSÉ) a sur le travail féminin un effet négatif en milieu rural et un effet positif en milieu urbain. L'effet positif de SSÉ sur la fécondité dans les régions rurales reflète la perpétuation des valeurs traditionnelles en milieu agricole. L'aide de la parenté (AIDE) est significative dans l'équation d'emploi mais non dans l'équation de fécondité.

La signification statistique des variables prédéterminées (EMPLAV, ENFDÉS) montre qu'une partie des décisions concernant le nombre d'enfants et la participation de l'épouse à la main-d'œuvre sont prises avant le mariage ou au moment du mariage. Elle est aussi caractéristique du fait que la fécondité désirée au mariage est positivement reliée à la variance effective de la fécondité : plus le nombre d'enfants désirés est élevé, plus le couple a de mal à réaliser ses projets de fécondité.

Les variables démographiques et familiales ont une contribution significative, allant en général dans le sens attendu. Par exemple, l'influence positive d'ORIG dans l'équation d'emploi des zones rurales montre que les femmes d'un certain âge acceptent plus facilement leur rôle traditionnel dans le secteur agricole. En outre, l'influence du mari semble importante. On note que l'origine rurale du mari est statistiquement significative dans les deux équations de fécondité, tandis que celle de la femme ne l'est pas.

Le principal résultat de cette étude est de faire apparaître un lien causal important entre l'emploi et la fécondité. Cette influence de l'emploi sur la fécondité s'exerce à la fois directement et indirectement à travers les attitudes des femmes 
TABLEAU 4-Résultats des estimations par la méthode généralisée des moindres carrés à deux étapes : régions urbaines

\begin{tabular}{|c|c|c|c|c|}
\hline \multicolumn{3}{|c|}{ Équations } & \multicolumn{2}{|c|}{ EMPLOI } \\
\hline Variables & Structure & Variance & Structure & Variance \\
\hline FÉCON & -1 & - & $\begin{array}{l}0,00849 \\
(0,03)\end{array}$ & - \\
\hline EMPL & $\begin{array}{c}-0,4175^{*} \\
(0,170)\end{array}$ & - & -1 & - \\
\hline RÔLSEX & $\begin{array}{l}1,3768^{*} \\
(0,338)\end{array}$ & - & $\begin{array}{c}0,15192 \\
(0,173)\end{array}$ & - \\
\hline \$LOISIR & $\begin{array}{l}-0,89164 * \\
(0,427)\end{array}$ & & $\begin{array}{l}2,3787^{*} \\
(0,218)\end{array}$ & \\
\hline REVFAM & $\begin{array}{l}0,36059 * \\
(0,095)\end{array}$ & $\begin{array}{c}47,552 * \\
(7,48)\end{array}$ & $\begin{array}{l}-0,70906^{*} \\
(0,084)\end{array}$ & $\begin{array}{l}4,5628^{*} \\
(0,398)\end{array}$ \\
\hline \$RELOISIR & - & $\begin{array}{c}134,39 \\
(14,2)\end{array}$ & $\begin{array}{l}1,2583^{*} \\
(0,176)\end{array}$ & $\begin{array}{l}5,3094 * \\
(0,76)\end{array}$ \\
\hline NPIĖCES & $\begin{array}{l}5,7304 * \\
(1,018)\end{array}$ & $\begin{array}{c}-689,39 * \\
(96,3)\end{array}$ & $\begin{array}{c}-1,2970^{*} \\
(0,447)\end{array}$ & \\
\hline SCOLAR & $\begin{array}{c}-2,5582^{*} \\
(1,20)\end{array}$ & & $\begin{array}{l}3,7808^{*} \\
(0,811)\end{array}$ & $\begin{array}{c}-55,785^{*} \\
(5,08)\end{array}$ \\
\hline SCOLARH & $\begin{array}{c}-0,95168 \\
(0,745)\end{array}$ & $\begin{array}{r}627,2^{*} \\
(68,6)\end{array}$ & $\begin{array}{c}-1,4192 \\
(1,256)\end{array}$ & \\
\hline SSÉ & $\begin{array}{c}0,3101 \\
(0,273)\end{array}$ & $\begin{array}{c}-409,34^{*} \\
(12,9)\end{array}$ & $\begin{array}{l}0,2088^{*} \\
(0,099)\end{array}$ & \\
\hline AIDE & $\begin{array}{l}-0,32283 \\
(4,75)\end{array}$ & $\begin{array}{c}4524,5^{*} \\
(385,0)\end{array}$ & $\begin{array}{l}7,839^{*} \\
(1,66)\end{array}$ & \\
\hline EMPLAV & - & & $\begin{array}{l}4,5796^{*} \\
(0,23)\end{array}$ & \\
\hline ENFDÉS & $\begin{array}{c}18,669^{*} \\
(2,69)\end{array}$ & $\begin{array}{c}3176,2 * \\
(314,0)\end{array}$ & - & \\
\hline ÂGE & - & & $\begin{array}{l}0,26187 \\
(0,157)\end{array}$ & \\
\hline
\end{tabular}

relativement aux rôles sexuels. La réciproque (influence de la fécondité sur l'emploi) ne ressort pas. Cela signifie que le travail de la femme peut influencer le nombre de ses enfants mais que sa féconditê n'influence pas sa participation à la main-d'œuvre. Avec d'autres données et un modèle légèrement différent, nous en étions arrivés aux mêmes conclusions pour la région métropolitaine d'Athènes (Magdalinos et Symeonidou, 1989).

Néanmoins, nos résultats diffèrent de ceux de plusieurs études plus anciennes (voir par exemple Smith-Lovin et Tickamyer, 1978; Kyriazis et Henripin, 1982; Klijzing et al., 1987), qui font ressortir la relation inverse. Il se peut que cette 
Fécondité et travail féminin en Grèce : un modèle micro-économique 301

TABLEAU 4 (suite)

\begin{tabular}{|c|c|c|c|c|}
\hline \multirow{2}{*}{$\begin{array}{l}\text { Équation } \\
\text { Variables }\end{array}$} & \multicolumn{2}{|c|}{ FÉCONDITÉ } & \multicolumn{2}{|c|}{ EMPLOI } \\
\hline & Structure & Variance & Structure & Variance \\
\hline DURMAR & $\begin{array}{l}1,6894^{*} \\
(0,453)\end{array}$ & $\begin{array}{c}828,79 * \\
(64,2)\end{array}$ & - & \\
\hline ÂGEMAR & $\begin{array}{c}-2,537^{*} \\
(0,457)\end{array}$ & & - & \\
\hline ORIG & $\begin{array}{c}-1,5877 \\
(4,17)\end{array}$ & & $\begin{array}{c}8,494^{*} \\
(2,38)\end{array}$ & $\begin{array}{c}-166,19^{*} \\
(14,7)\end{array}$ \\
\hline TRAVME் & - & $\begin{array}{r}-4344,4^{*} \\
(368,0)\end{array}$ & $\begin{array}{l}0,82106 \\
(2,85)\end{array}$ & $\begin{array}{l}139,67^{*} \\
(10,4)\end{array}$ \\
\hline ENFMĖ & $\begin{array}{l}2,9149^{*} \\
(1,08)\end{array}$ & & - & $\begin{array}{c}-25,379^{*} \\
(4,38)\end{array}$ \\
\hline SCOLPE & - & $\begin{array}{c}-721,82^{*} \\
(53,9)\end{array}$ & - & $\begin{array}{c}22,33^{*} \\
(2,4)\end{array}$ \\
\hline SSÉPE் & - & & - & $\begin{array}{c}-3,3515^{\circ} \\
(0,74)\end{array}$ \\
\hline VETOH & - & & $\begin{array}{r}-26,46 * \\
(3,08)\end{array}$ & \\
\hline ORIGH & $\begin{array}{c}11,724^{*} \\
(5,33)\end{array}$ & $\begin{array}{c}7176,9^{*} \\
(423,0)\end{array}$ & $\begin{array}{l}2,5166 \\
(1,97)\end{array}$ & \\
\hline ENFMËH & $\begin{array}{c}7,769^{*} \\
(1,14)\end{array}$ & $\begin{array}{r}-1367,4^{*} \\
(94,1)\end{array}$ & - & \\
\hline Constante & 0,2201 & $-1,0951$ & 0,52217 & 0,82341 \\
\hline $\mathrm{R}^{2}$ & 0,868 & 0,965 & 0,764 & 0,870 \\
\hline $\mathrm{R}^{2}$ ajusté & 0,866 & 0,964 & 0,761 & 0,869 \\
\hline$s^{2}$ & 0,945 & 3,76 & 17,66 & 2,89 \\
\hline $\mathrm{F}$ & 563,54 & 3446,9 & 251,29 & 1271,04 \\
\hline Observations & 1388 & 1532 & 1259 & 1532 \\
\hline
\end{tabular}

NOTE * : significatif au seuil de $5 \%$; entre parenthèses, les écarts types. $R^{2}, s^{2}, F$ : voir le tableau 3. Tirets : restrictions a priori; cases vides (équations de variance) : restrictions at posteriori. Nous avons exclu les observations pour lesquelles la variance estimée était. inférieure à $1 \%$ de la variance initiale.

divergence soit due à la différence entre les échantillons plutôt qu'à une différence entre les modèles ou les techniques d'esti-. mation. Les décisions relatives à la taille de la famille mettent des années à se concrétiser, de sorte que le rapport de causalité entre la fécondité et l'emploi est lié à des décisions prises au début du mariage ou à chaque accroissement de la parité. L'un des principaux facteurs qui influencent cette décision est le coût qu'assument les femmes chaque fois qu'elles décident de quitter et de reprendre le travail. 
TABLEAU 5 - Résultats des estimations par la méthode généralisée des moindres carrés à deux étapes : régions rurales

\begin{tabular}{|c|c|c|c|c|}
\hline \multicolumn{3}{|c|}{ Équations } & \multicolumn{2}{|c|}{ EMPLOI } \\
\hline Variables & Structure & Variance & Structure & Variance \\
\hline FÉCON & -1 & - & $\begin{array}{l}0,04187 \\
(0,025)\end{array}$ & - \\
\hline EMPL & $\begin{array}{c}-0,4000^{*} \\
(0,129)\end{array}$ & - & -1 & - \\
\hline RÔLSEX & $\begin{array}{l}1,0529 * \\
(0,415)\end{array}$ & - & $\begin{array}{l}0,41071^{*} \\
(0,165)\end{array}$ & - \\
\hline \$LOISIR & $\begin{array}{c}-1,8592^{*} \\
(0,451)\end{array}$ & & $\begin{array}{c}-0,34192 \\
(0,785)\end{array}$ & \\
\hline REVFAM & $\begin{array}{l}0,34071^{*} \\
(0,113)\end{array}$ & & $\begin{array}{c}0,15474 \\
(0,114)\end{array}$ & $\begin{array}{l}6,4146^{*} \\
(0,864)\end{array}$ \\
\hline \$RELOISIR & - & & $\begin{array}{l}0,7514^{*} \\
(0,14)\end{array}$ & $\begin{array}{l}8,7462 \text { * } \\
(1,21)\end{array}$ \\
\hline NPIĖCES & $\begin{array}{l}4,3289 * \\
(1,24)\end{array}$ & $\begin{array}{c}-666,79^{*} \\
(83,1)\end{array}$ & $\begin{array}{c}-1,6069^{*} \\
(0,788)\end{array}$ & $\begin{array}{l}-43,540^{*} \\
(10,2)\end{array}$ \\
\hline SCOLAR & $\begin{array}{c}-6,2701^{*} \\
(1,44)\end{array}$ & $\begin{array}{c}295,55^{*} \\
(91,5)\end{array}$ & $\begin{array}{l}3,4861 \text { * } \\
(0,83)\end{array}$ & $\begin{array}{c}38,816^{*} \\
(9,39)\end{array}$ \\
\hline SCOLARH & $\begin{array}{l}0,20789 \\
(0,872)\end{array}$ & $\begin{array}{c}-162,51^{*} \\
(62,9)\end{array}$ & $\begin{array}{c}-1,4396^{*} \\
(0,314)\end{array}$ & \\
\hline SSÉ & $\begin{array}{l}0,82361^{*} \\
(0,235)\end{array}$ & & $\begin{array}{c}-0,63591^{*} \\
(0,165)\end{array}$ & $\begin{array}{l}-8,9041^{*} \\
(2,03)\end{array}$ \\
\hline AIDE & $\begin{array}{l}4,5335 \\
(4,14)\end{array}$ & & $\begin{array}{l}6,3185^{*} \\
(1,59)\end{array}$ & \\
\hline EMPLAV & - & & $\begin{array}{l}2,6793^{*} \\
(0,19)\end{array}$ & \\
\hline ENFDÉS & $\begin{array}{c}32,734 * \\
(2,49)\end{array}$ & $\begin{array}{c}1932,9^{*} \\
(354,0)\end{array}$ & - & \\
\hline ÂGE & - & & $\begin{array}{l}0,69938^{*} \\
(0,135)\end{array}$ & \\
\hline
\end{tabular}

En Grèce, ce coût est très élevé. Il est rare que les conditions de travail couvrent adéquatement les besoins des femmes; les congés de maternité prolongés et le travail à temps partiel ne sont pas organisés. En outre, il devient de plus en plus difficile avec l'âge de trouver du travail, et la complexité des régimes de retraite et des programmes d'avantages sociaux rend le transfert de droits très difficile. C'est pourquoi, sans doute, les Grecques tendent ou bien à garder leur emploi ou bien à le quitter définitivement. Le tableau 6 présente une comparaison entre notre échantillon et l'échantillon étudié par Ni Bhrolchain (1980) en Angleterre. 
Fécondité et travail féminin en Grèce : un modèle micro-économique 303

TABLEAU 5 (suite)

\begin{tabular}{|c|c|c|c|c|}
\hline \multirow[t]{2}{*}{ Équation } & \multicolumn{2}{|c|}{ FÉCONDTTÉ } & \multicolumn{2}{|c|}{ EMPLOI } \\
\hline & Structure & Variance & Structure & Variance \\
\hline DURMAR & $\begin{array}{l}3,5933^{*} \\
(0,5)\end{array}$ & $\begin{array}{c}570,73^{*} \\
(54,9)\end{array}$ & - & \\
\hline ĀGEMAR & $\begin{array}{c}0,46472 \\
(0,483)\end{array}$ & & - & \\
\hline ORIG & $\begin{array}{c}-1,033 \\
(7,4)\end{array}$ & $\begin{array}{c}4816,3^{*} \\
(569,0)\end{array}$ & $\begin{array}{c}-2,5628 \\
(3,15)\end{array}$ & \\
\hline TRAVMÈ & - & & $\begin{array}{c}22,421 * \\
(3,01)\end{array}$ & $\begin{array}{c}2,00,67 * \\
(45,7)\end{array}$ \\
\hline ENFMÈ & $\begin{array}{l}3,3218^{*} \\
(1,29)\end{array}$ & $\begin{array}{c}1084,6^{*} \\
(131,0)\end{array}$ & - & \\
\hline SCOLPE & - & $\begin{array}{c}-786,73^{*} \\
(51,6)\end{array}$ & - & \\
\hline SSÉPÈ & 一 & & - & $\begin{array}{c}-15,471^{*} \\
(4,75)\end{array}$ \\
\hline VETOH & - & $\begin{array}{r}-4628,9^{*} \\
(832,0)\end{array}$ & $\begin{array}{c}-47,198^{*} \\
(4,25)\end{array}$ & $\begin{array}{c}-241,46^{*} \\
(56,1)\end{array}$ \\
\hline ORIGH & $\begin{array}{c}87,284^{*} \\
(9,23)\end{array}$ & $\begin{array}{r}-6157,4^{*} \\
(354,0)\end{array}$ & $\begin{array}{c}-6,2162 \\
(3,95)\end{array}$ & \\
\hline ENFMEH & $\begin{array}{l}4,0837^{*} \\
(1,13)\end{array}$ & $\begin{array}{l}936,96^{*} \\
(157,0)\end{array}$ & - & \\
\hline Constante & $-0,13184$ & $-1,6743$ & $-2,1264$ & 0,52907 \\
\hline $\mathrm{R}^{2}$ & 0,903 & 0,842 & 0,841 & 0,386 \\
\hline $\mathrm{R}^{2}$ ajusté & 0,902 & 0,841 & 0,839 & 0,383 \\
\hline $\mathrm{s}^{2}$ & 0,505 & 7,56 & 3,94 & 1,91 \\
\hline $\mathrm{F}$ & 946,11 & 801,51 & 531,8 & 128,62 \\
\hline Observations & 1646 & 1661 & 1630 & 1664 \\
\hline
\end{tabular}

NOTE * : significatif au seuil de $5 \%$; entre parenthèses, les écarts types. $\mathrm{R}^{2}, \mathrm{~s}^{2}, \mathrm{~F}$ : voir le tableau 3. Tirets : restrictions a priori; cases vides (équations de variance) : restrictions a posteriori. Nous avons exclu les observations pour lesquelles la variance estimée était inférieure à $1 \%$ de la variance initiale.

Bien que les deux échantillons ne soient pas directement comparables, il est manifeste que le marché du travail n'est pas le même en Angleterre et en Grèce, où il se caractérise par un manque de flexibilité. Le lien entre la fécondité et le travail n'est pas significatif au plan statistique à cause de ce qu'il en coûte pour quitter ou reprendre le travail en raison de cette rigidité du système.

L'effet positif et statistiquement significatif du coefficient des attitudes relatives aux rôles sexuels dans l'équation de 
TABLEAU 6 - Présence des femmes mariées a sur le marché du travail en rapport avec le mariage et la première naissance, selon le lieu de résidence (\%)

\begin{tabular}{|c|c|c|c|c|c|}
\hline & thènes & $\begin{array}{l}\text { Autres } \\
\text { régions } \\
\text { urbaines }\end{array}$ & $\begin{array}{l}\text { Régions } \\
\text { rurales }\end{array}$ & Grèce & $\begin{array}{l}\text { Angle- } \\
\text { terre }\end{array}$ \\
\hline Taille de l'échantillon (N) & 1881 & 2182 & 2275 & 6338 & 5321 \\
\hline Ont toujours travaillé & 17,4 & 12,7 & 19,0 & 16,8 & 11 \\
\hline $\begin{array}{l}\text { Ont quitté leur emploi } \\
\text { en se mariant }\end{array}$ & 22,3 & 22,0 & 25,4 & 23,4 & 36 \\
\hline $\begin{array}{l}\text { - Et ont repris le travail } \\
\text { au moins une fois b }\end{array}$ & 35,2 & 34,5 & 51,7 & 40,0 & 64 \\
\hline $\begin{array}{l}\text { Ont quitté leur emploi } \\
\text { à la première naissance }\end{array}$ & 14,3 & 9,2 & 6,2 & 10,7 & 41 \\
\hline $\begin{array}{l}\text { - Et ont repris le travail } \\
\text { au moins une fois b }\end{array}$ & 35,2 & 37,1 & 67,3 & 43,2 & 65 \\
\hline
\end{tabular}

Source : Symeonidou, 1990; données inédites de l'Enquête nationale sur la féconditê.

a. Échantillon constitué de toutes les femmes "fécondes" et non sous-échantillon utilisé pour calculer le système d'équations.

b. Par rapport aux pourcentages de la ligne précédente.

fécondité est une caractéristique particulièrement intéressante de notre modèle. Il corrobore nos résultats antérieurs pour la région d'Athènes (Smith-Lovin et Tickamyer, 1978, obtiennent le même effet positif, mais non significatif). Il peut paraître surprenant que des femmes aux attitudes plus "émancipées" tendent à avoir plus d'enfants, d'autant que les données brutes attestent une corrélation négative fortement significative entre ces attitudes et la fécondité. Le coefficient de corrélation simple est de $-0,18$ et le coefficient de corrélation partielle, une fois isolée l'influence des variables exogènes, est de $-0,08$. Dans tous les cas, le niveau de signification est inférieur à $0,1 \%$. Il semble que cet effet positif soit produit par la structure que nous avons imposée aux données.

Notre modèle ne prend pas explicitement en compte le coût des enfants en temps. Néanmoins, les femmes aux opinions plus traditionnelles accordent une importance plus grande aux responsabilités maternelles et, après avoir eu un ou deux enfants, elles peuvent se sentir obligées de combler les attentes normatives traditionnelles liées à l'éducation des enfants. De plus, ces femmes sont moins portées à demander l'aide de leur mari ou à tirer tout le parti possible des services de garde 
existants. En conséquence, si la femme remplit ses propres exigences normatives, la variable rôles sexuels joue dans l'équation de fécondité le rôle d'une variable de coût puisqu'elle est en raison inverse de la quantité de travail requise par la responsabilité maternelle.

À un autre point de vue, l'effet positif sur la fécondité des attitudes relatives aux rôles sexuels témoigne d'une évolution sociale intéressante. Si nous supposons une augmentation de l'emploi par bonds de $10 \%$, chaque bond serait directement la cause d'une diminution de la fécondité de 4,18 dans les régions urbaines et de 4,0 et dans les régions rurales. Cependant, la même augmentation de l'emploi entraînerait indirectement un renforcement des attitudes plus féministes, soit un déplacement de 0,84 à chaque bond. Cela implique une augmentation indirecte de la fécondité de 1,16 par bond dans les régions urbaines et de 0,89 dans les régions rurales. L'effet global serait une diminution de la fécondité de 3,02 dans les régions urbaines et de 3,11 dans les régions rurales. Si l'on fait intervenir l'influence des attitudes liées aux rôles sexuels, l'effet direct diminue de $28 \%$ dans les villes et de $22 \%$ en territoire rural. Prises globalement, nos données montrent que l'effet négatif final de la participation accrue des femmes au marché du travail sur leur fécondité est vraisemblablement moins grand que l'effet qui ressort de l'analyse de corrélation simple.

\section{COMPARAISONS ET CONCLUSIONS}

Notre analyse révèle qu'il existe des différences entre les régions au plan de l'emploi et de la fécondité. Les tableaux 1 et 6 illustrent ces différences pour ce qui est du travail féminin et le tableau 7 pour la fécondité réalisée, désirée et jugée "idéale". Le nombre moyen de naissances vivantes est plus faible à Athènes, plus élevé dans les régions rurales et se situe à un niveau intermédiaire dans les autres régions urbaines. Il en va de même pour la taille désirée et la taille idéale de la famille. Cela implique que les équations de comportement ne seront pas les mêmes dans les divers milieux de vie.

Pour resserrer la comparaison, nous avons donc recalculé les équations de fécondité et d'emploi de la région d'Athènes en utilisant ces nouvelles variables. Les coefficients des équations de fécondité et d'emploi suivant la région sont présentés aux tableaux 8 et 9 . 
TABLEAU 7 - Taille moyenne de la famille : réelle, désirée et "idéale", selon le lieu de résidence ${ }^{\text {a }}$

\begin{tabular}{lcccc}
\hline $\begin{array}{l}\text { Taille de la famille } \\
\text { (nombre d'enfants) }\end{array}$ & $\begin{array}{c}\text { Région } \\
\text { d'Athènes }\end{array}$ & $\begin{array}{c}\text { Autres régions } \\
\text { urbaines }\end{array}$ & $\begin{array}{c}\text { Rẽgions } \\
\text { rurales }\end{array}$ & Grèce \\
\hline Réelle & 1,75 & 1,97 & 2,21 & 1,98 \\
Désirée & 2,16 & 2,27 & 2,40 & 2,29 \\
Jugée idéale & 2,55 & 2,70 & 2,84 & 2,70 \\
Taille de l'échantillon & 1881 & 2164 & 2259 & 6315 \\
\hline
\end{tabular}

Source : Symeonidou, 1990; données inédites de l'Enquête nationale sur la fécondité.

a. Le nombre d'enfants désiré est la somme des enfants qu'a dêjà la répondante et de ceux qu'elle dit souhaiter avoir encore; le nombre d'enfants idéal est fourni par les réponses à la question suivante : à votre avis, quel est le nombre idéal d'enfants pour la famille grecque moyenne?

TABLEAU 8 - Équation d'estimation de la fécondité, selon le lieu de résidence

\begin{tabular}{|c|c|c|c|c|c|}
\hline & Athènes & & $\begin{array}{c}\text { Autres régions } \\
\text { urbaines }\end{array}$ & & $\begin{array}{l}\text { Régions } \\
\text { rurales }\end{array}$ \\
\hline EMPL & $-0,5218^{*}$ & & $-0,4175^{*}$ & & $-0,4000^{*}$ \\
\hline RÔLSEX & $2,327^{*}$ & $\dagger$ & $1,3768^{*}$ & & $1,0529 *$ \\
\hline \$LOISIR & $-0,50252^{*}$ & & $-0,89164^{*}$ & & $-1,8592^{*}$ \\
\hline REVFAM & $0,27660^{*}$ & & $0,36095^{*}$ & & $0,34071^{*}$ \\
\hline NPIECES & $3,2657^{*}$ & & $5,7304^{*}$ & & $4,3289^{*}$ \\
\hline SCOLAR & $-4,9521^{*}$ & & $-2,5582^{*}$ & $\dagger$ & $-6,2701^{*}$ \\
\hline SCOLARH & $-0,0556$ & & $-0,95268$ & & 0,20798 \\
\hline $\mathrm{SSE}$ & 0,0579 & & 0,31010 & & $0,82361^{*}$ \\
\hline AIDE & 0,0236 & & $-0,3283$ & & 4,5335 \\
\hline ENFDES & $12,883^{*}$ & & $18,669^{*}$ & $\dagger$ & $32,734^{*}$ \\
\hline DURMAR & $2,0421^{*}$ & & $1,6894^{*}$ & $\dagger$ & $3,5933^{*}$ \\
\hline ÂGEMAR & $-1,583^{*}$ & & $-2,537^{*}$ & + & 0,46472 \\
\hline ORIG & $-0,5365$ & & $-1,5877$ & & $-1,0330$ \\
\hline ENFMĖ & $3,4231^{*}$ & & $2,9149^{*}$ & & $3,3218^{*}$ \\
\hline ORIGH & $32,248^{*}$ & $\dagger$ & $11,724^{*}$ & $\dagger$ & $87,284^{*}$ \\
\hline ENFMĖH & 2,8114 & $\dagger$ & $7,7694^{*}$ & $\dagger$ & $4,0837^{*}$ \\
\hline Constante & 0,3768 & & 0,2201 & & $-0,13184$ \\
\hline $\mathrm{R}^{2}$ ajustê & 0,810 & & 0,866 & & 0,902 \\
\hline F & 350,4 & & 563,5 & & 946,1 \\
\hline
\end{tabular}

* significatif au seuil de $5 \%$; $\dagger$ différences statistiquement significatives (même seuil). 
TABLEAU 9-Équation d'estimation de l'emploi, selon le lieu de résidence

\begin{tabular}{|c|c|c|c|c|c|}
\hline & Athènes & & $\begin{array}{c}\text { Autres régions } \\
\text { urbaines }\end{array}$ & & $\begin{array}{l}\text { Régions } \\
\text { rurales }\end{array}$ \\
\hline FÉCON & $-0,0305$ & & 0,00849 & & 0,04187 \\
\hline RÔLSEX & 0,0283 & & 0,15192 & & $0,41071^{*}$ \\
\hline SLOISIR & $2,6057^{*}$ & & $2,3787^{*}$ & $\dagger$ & $-0,34192$ \\
\hline REVFAM & $-0,6031^{*}$ & & $-0,70906^{*}$ & $\dagger$ & 0,15474 \\
\hline \$RELOISIR & $1,6403^{*}$ & & $1,2583^{*}$ & $\dagger$ & $0,75140^{*}$ \\
\hline NPIECEES & $-1,1650^{*}$ & & $-1,2970^{*}$ & & $-1,6069^{*}$ \\
\hline SCOLAR & $1,8891^{*}$ & & $3,7808^{*}$ & & $3,4861^{*}$ \\
\hline SCOLARH & 0,2832 & & $-1,4192$ & & $-1,4396^{*}$ \\
\hline SSÉ & $-0,0173$ & $\dagger$ & $0,2088^{*}$ & $\dagger$ & $-0,63591^{*}$ \\
\hline AIDE & $8,3595^{*}$ & & $7,8390^{*}$ & & 6,3185 \\
\hline EMPLAV & $1,7586^{*}$ & $\dagger$ & $4,5796^{*}$ & $\dagger$ & $2,6793^{*}$ \\
\hline ÃGE & 0,14310 & & 0,26187 & $\dagger$ & $0,69938^{*}$ \\
\hline ORIG & $-0,8993$ & $\dagger$ & $8,4940^{*}$ & $\dagger$ & $-2,5628$ \\
\hline TRAVMÉ & 1,1750 & & 0,82106 & $\dagger$ & $22,421^{*}$ \\
\hline VETOH & $-27,391^{*}$ & $\dagger$ & $-26,460^{*}$ & $\dagger$ & $-47,198^{*}$ \\
\hline ORIGH & $-0,6031$ & & 2,5166 & & $-4,2162$ \\
\hline Constante & 0,2560 & & 0,52217 & & $-2,1264$ \\
\hline $\mathrm{R}^{2}$ ajusté & 0,632 & & 0,761 & & 0,839 \\
\hline F & 131,1 & & 251,3 & & 531,8 \\
\hline
\end{tabular}

* Significatif au seuil de $5 \%$; différences statistiquement significatives (même seutl).

Toutefois, on l'a vu, les variables REVSÉP (revenu familial sans le salaire de l'épouse) et \$OPT (coût d'option du travail) de notre étude précédente (Magdalinos et Symeonidou, 1989) ont été remplacées ici par \$LOISIR (coût du loisir), REVFAM (revenu familial total) et \$RELOISIR (coût relatif du loisir).

Le tableau 8 montre que l'équation de fécondité ne fait pas ressortir beaucoup de différences statistiquement significatives entre Athènes et les autres zones urbaines ${ }^{6}$. L'effet positif plus important de RÔLSEX sur FÉCON à Athènes était attendu, car le temps nécessaire pour l'uélevage" traditionnel y est plus long que dans les autres régions. L'explication des différences statis-

6 Soit $\beta_{1}$ et $\beta_{2}$, les coefficients de la mème variable dans deux régions différentes, et $\sigma_{1}$ et $\sigma_{2}$, les écarts types respectifs. Comme les échantillons de deux régions différentes sont indépendants, la différence $\beta_{1}-\beta_{2}$ est statistiquement significative au seuil de $5 \%$ si

$$
\left|\beta_{1}-\beta_{2}\right| / \sqrt{\sigma_{1}^{2}+\sigma_{2}^{2}}>1,96 \text {. }
$$


tiquement significatives que présentent les coefficients des variables ORIGH et ENFMËH est moins évidente. Si l'on compare la ville et les régions rurales, on se rend compte que l'effet négatif de l'instruction des femmes sur la fécondité est plus marqué dans les secondes : les femmes instruites réagissent plus fortement aux valeurs traditionnelles qui prévalent dans ces régions. L'effet positif de ENFDÉS est plus marqué dans les régions rurales que dans les régions urbaines. Il semble que les gens trouvent plus facile de réaliser leur désir d'avoir une grande famille en milieu rural. Dans le même ordre d'idées, le plus grand effet positif de DURMAR sur la fécondité dans les régions rurales et le fait que ÂGEMAR n'y soit pas significatif impliquent que les femmes y ont une plus longue période de reproduction.

En ce qui concerne l'équation d'emploi, on constate, encore ici, qu'il y a peu de différences statistiquement significatives entre Athènes et les autres régions urbaines. Les différences sont plus nombreuses entre les villes et le milieu rural, où, en particulier, les variables économiques \$LOISIR, REVFAM et \$RELOISIR ont une influence beaucoup plus forte. Cela peut s'expliquer par le fait que, dans les campagnes, beaucoup de femmes travaillent sans salaire au sein de l'entreprise familiale. Cette structure d'emploi traditionnelle est moins sensible aux considérations économiques. Eu égard à la variable SSÉ, nous constatons que dans les régions urbaines hors d'Athènes, les femmes à statut supérieur sont plus souvent présentes dans la main-d'œuvre qu'à Athènes; mais SSÉ a un effet négatif dans les régions rurales, ce qui peut signifier que les femmes à statut plus élevé sont moins disposées à travailler dans le secteur agricole.

L'effet de la variable EMPLAV est plus net dans les autres régions urbaines qu'à Athènes ou dans les régions rurales. Il se peut que les femmes concernées passent par une étape transitoire d'indépendance économique dont on ne voit guère la trace dans les zones rurales, où, encore une fois, les femmes travaillent habituellement sans salaire dans l'entreprise familiale. L'effet de EMPLAV est encore moindre à Athènes, où les rôles de mère et de travailleuse sont plus difficilement compatibles. Des raisons similaires peuvent expliquer qu'ORIG n'ait pas les mêmes effets à Athènes que dans les autres régions urbaines et dans les régions rurales. TRAVMÈ exerce une très forte influence sur l'emploi des femmes dans les régions rurales, où la tradition de travailler dans l'entreprise familiale semble se 
transmettre de mère en fille, alors qu'elle n'a pas d'effet à Athènes et dans les autres zones urbaines. Enfin, comme on s'y attendait, l'attitude négative du mari envers le travail de l'épouse a sur le travail féminin un effet négatif beaucoup plus fort dans les zones rurales que dans les zones urbaines.

Notre modèle peut sans aucun doute être exploité pour la. prédiction, l'analyse de politiques et l'évaluation. Une description complète de ses possibilités dépasserait le cadre de cet article, mais nous allons tout de même dégager les conséquences pratiques de certains résultats en termes politiques.

La fécondité diminue de façon continue en Grèce depuis trois décennies. Le taux brut de natalité est passé de 18,9 naissances pour mille en 1960 à 15,4 en 1980 et à 10,1 en 1992 . En outre, depuis 1981, le nombre moyen d'enfants par femme en âge de se reproduire est descendu sous le seuil de remplacement des générations (2,1 enfants par femme). Le dernier chiffre disponible pour 1992 est 1,41. Simultanément, la proportion de personnes de 65 ans et plus est passée de $8 \%$ en 1961 à 14,2 \% en 1991 (Eurostat, 1992; NSSG, 1985). Selon les projections actuelles, la population de la Grèce commencera à diminuer à la fin de la présente décennie (Tziafetas et Tzougas, 1988).

Compte tenu des données présentées dans cet article, nous reprenons les mesures proposées dans l'étude précédemment consacrée à Athènes comme moyen de stimuler la fécondité (Magdalinos et Symeonidou, 1989).

i) Une modernisation $d u$ marché du travail. Notre modèle montre en premier lieu qu'une amélioration des conditions de travail (mise en place d'emplois à temps partiel, de congés. de maternité prolongés entièrement ou partiellement payés, réinsertion plus facile sur le marché du travail) est susceptible de faire augmenter la fécondité. Dans le secteur public, en particulier, les femmes devraient être assurées de retrouver, à la fin de leur congé, non seulement leur emploi mais aussi leur plein statut. Cette flexibilité permettrait une réduction du taux d'activité au cours de la période critique de l'arrivée des enfants. Le caractère statique de notre modèle ne permet pas d'en tirer plus d'informations à cet égard, mais il est certain que les effets d'une adaptation du marché du travail méritent plus ample examen.

ii) Une politique de revenu. Une augmentation du revenu des familles, surtout des plus pauvres, aurait un effet positif sur la fécondité, à condition de ne pas accroître le coût du loisir, c'est- 
à-dire le salaire réel ou attendu de l'épouse. Ce type de politique aurait plus de chances de succès dans les régions rurales, car dans les zones urbaines une augmentation de REVFAM accentuerait la variance de la fécondité et la probabilité d'un échec de la mesure.

iii) Une politique visant à diminuer le coût des enfants. Si l'on pose que le coût direct réel des enfants a le même effet que son substitut (NPIĖCES); une diminution de ce coût augmenterait le niveau moyen de la fécondité tout en réduisant sa variance. Ces deux effets seraient plus marqués dans les régions urbaines. Notamment, un programme de logement conçu pour les jeunes couples pourrait accroître la fécondité à la fois directement, en augmentant NPIÈCES, et, puisque le logement est l'un des principaux problèmes que doivent affronter les jeunes couples urbains, indirectement, en réduisant ÂGEMAR.

iv) Une campagne d'opinion. Le nombre d'enfants désiré par un couple au moment du mariage (ENFDÉS) est influencé par les valeurs de la société, qui peuvent elles-mêmes être influencées par une campagne de promotion des familles nombreuses. Cette politique, tout en étant la moins coûteuse à adopter, n'est pas la plus prometteuse, car une augmentation de la valeur de ENFDÉS provoquerait une immense augmentation de la variance.

Ces suggestions ne suffisent évidemment pas à constituer un programme, mais elles donnent une idée des applications potentielles de notre modèle. Avant de pouvoir formuler une politique efficace et complète, il y aurait lieu de mener une analyse stochastique coûts-bénéfices, en particulier sur des sousgroupes et sur des cohortes d'âge au sein de la population, en utilisant des versions adaptées du modèle.

\section{RÉFÉRENCES BIBLIOGRAPHIQUES}

AMEMIYA, T., 1977. "A Note on Heteroscedastic Model", Journal of Econometrics, $6: 365-370$; et "Corrigenda", $8: 275$.

BREUSCH, T. S., et A. R. PAGAN, 1979. "A Simple Test for Heteroscedasticity and Random Coefficient Variation", Econometrica, 47 : 1287-1294.

BHROLCHAIN, M. Ni, 1980. Fertility and Female Employment. What Relationship? London School of Hygiene and Tropical Medicine, Center for Population Studies, Working Paper No. 80-3.

CARTER, R. A., et A.L. NAGAR, 1977. "Coefficients of Correlation for Simultaneous Equation Systems", Journal of Econometrics, 6 : 39-50. 
EUROSTAT, 1992. Demographic Statistics. Luxembourg.

HARVEY, A. C., 1976. "Estimating Regression Models with Multiplicative Heteroscedasticity", Econometrica, 44 : 461-465.

KLIJZING, E., J. SIEGERS, N. KEILMAN et L. GROOT, 1987. "Static Versus Dynamic Analysis of the Interaction between Female Labor Force Participation and Fertility", European Journal of Fopulation, $4: 97-116$.

KYRIAZIS, N., et J. HENRIPIN, 1982. "Women's Employment and Fertility in Quebec", Population Studies, 36 : 431-440.

LEAMER, E. E., 1988. "3 Things That Bother Me", The Economic Record, $18: 331-335$.

MAGDALINOS, M., et H. SYMEONIDOU, 1989. "Modeling the FertilityEmployment Relationship: Simultaneity and Misspecification Testing", European Journal of Population, 5 : 119-143.

MROZ, T. A., 1987. "The Sensitivity of an Empirical Model of Married Women's Hours of Work to Economic and Statistical Assumptions", Econometrica, 55 : 765-799.

NSSG (National Statistical Service of Greece), 1985. Demographic Yearbook. Athènes

OLSEN, R. J., 1980. "A Least Squares Correction for Selectivity Bias", Econometrica, $48: 1815-1820$.

ROBINSON, P. M., 1987. "Asymptotically Efficient Estimation in the Presence of Heteroscedasticity of Unknown Form", Econometrica, 55 : 875-891.

SARGAN, J.D., 1958. "The Estimation of Economic Relationships Using Instrumental Variables", Econometrica, $26: 393-415$.

SCHULTZ, T. P., 1969. "An Economic Model of Family Planning and Fertility", Journal of Political Economy, 2 : 153-180.

SMITH-LOVIN, L., et A. R. TICKAMYER, 1978. "Nonrecursive Models of Labor Force Participation Fertility Behavior and Sex Role Attitudes", American Sociological Review, 43 : 541-557.

SYMEONIDOU, H., 1990. Fertility and Employment of Women in the Greater Athens Area. Athènes, The National Center of Social Research.

TREIMAN, D., 1977. Occupational Prestige in Comparative Perspective. New York, Academic Press Inc.

TZIAFETAS, G., et J. TZOUGAS, 1988. "Prospects for the Greek Population in the Light of the New Data about Vital Statistics". Athènes, The European Demographic Community, EDIM : 51-81.

WHITE, H., 1980. "A Heteroscedasticity-Consistent Covariance Matrix Estimator and a Direct Test for Heteroscedasticity", Econometrica, $48,4: 817-838$. 


\section{RÉSUMÉ - ABSTRACT - RESUMEN}

\section{SYMEONIDOU Hariclia et MAGDALINOS Michael - FÉCONDITÉ ET TRAVAIL FÉMININ EN GRĖCE : UN MODĖLE MICRO-ÉCONOMIQUE}

Magdalinos et Symeonidou (1989) ont étudié la relation entre la fécondité et l'emploi chez les femmes de la région métropolitaine d'Athènes. L'analyse présentée ici porte sur le reste de la Grèce. Elle fait appel à un échantillon de 3193 femmes mariées comprenant 1532 femmes des régions urbaines (à l'exclusion d'Athènes) et 1661 femmes des régions rurales. Un test de détection des erreurs de spécification a mis en évidence des différences de structure entre les deux univers ainsi que l'hétéroscédasticité de l'équation d'offre pour le travail et de l'équation de demande pour les enfants. Un dernier modèle composé de cinq équations de structure et de quatre équations de variance a été estimé. Ces nouveaux résultats confirment les précédents : l'emploi influence la fécondité directement et à travers les attitudes liées aux rôles féminins. On ne note aucune influence directe significative de la fécondité sur l'emploi, mais dans les zones rurales on observe un effet indirect significatif, par l'intermédiaire des attitudes liées aux rôles sexuels. Comme prévu, les équations de fécondité et d'emploi présentent des différences significatives entre zones urbaines et zones rurales.

\section{SYMEONIDOU Hariclia and MAGDALINOS Michael - A MICRO-ECONOMIC MODEL FOR THE FERTILITY-EMPLOYMENT RELATIONSHIP IN GREECE}

Magdalinos and Symeonidou (1989) examined the relationship between fertility and the employment of women in the Greater Athens Area. The study presented by them extends the analysis to the rest of Greece using a sample of 3,193 married women of which 1,532 lived in urban areas other than Athens and 1,661 women lived in rural areas. Misspecification testing revealed structural differences between urban and rural areas and heteroscedasticity in the supply equation for work and the demand equation for children. A final model consisting of five structural and four variance equations was estimated. The new results confirm earlier findings: employment influences fertility both directly as well as through women's sex-role attitudes. While no significant direct influence of fertility on employment was found, a significant indirect influence was found in the rural areas, as a result of attitudes towards sexual roles. As expected, significant differences exist in the fertility and employment equations between the urban and rural areas.

\section{SYMEONIDOU Hariclia y MAGDALINOS Michael - FECUNDIDAD Y TRABAJO FEMENINO EN GRECLA: MODELO MICRO-ECONÓMICO}

Magdalinos y Symeonidou (1989) estudiaron la relación entre fecundidad y empleo de las mujeres de la región metropolitana de Atenas. El análisis que aquí se presenta se extiende al resto de Grecia; se utilizó un muestrario de 3193 mujeres casadas, de las cuales 1532 viven en regiones urbanas (otras que Atenas) y 1661 en regiones rurales. Una prueba de detección de errores de especificación reveló ciertas diferencias de estructura entre ambas regiones (urbana y rurall, así como la heteroscedasticidad de la ecuación de oferta de trabajo y la de demanda de hijos. Se hizo la estimación de un último modelo compuesto por cinco ecuaciones de estructura y cuatro de variancia. Los nuevos resultados confirman los anteriores, a saber que la fecundidad está influenciada por el empleo, directamente y a través de las actitudes sobre las funciones de las mujeres. No se nota ningún efecto directo significativo de la fecundidad sobre el empleo pero, en las zonas rurales, se observa un efecto indirecto significativo, por medio de las actitudes hacia las funciones sexuales. Tal como se esperaba, las ecuaciones de fecundidad y de empleo presentan diferencias significativas entre las zonas urbanas y las zonas rurales. 